\title{
Research on the Layout of National Economic Mobilization Logistics Centers
}

\author{
Pingzhou Tang \\ North China Electric Power University/School of Economics and Management, Beijing, China \\ tangpingzhou@163.com \\ Huiying Tian \\ North China Electric Power University/School of Economics and Management, Beijing, China \\ thyweb@163.com
}

\begin{abstract}
The problem of the layout of NEM LC (National E conomic M obilization L ogistics Center) is one of the most important long-term decision-making issues. The result of NEMLC's layout directly impacts many aspects of mobilization, such as time, reliability, quality, efficiency, cost, and so on, consequently affects the effect of the mobilization. Reasonable NEM LC layout can bring people convenience in the daily life, reduce costs, and improve service efficiency and competitiveness. Poor NE M L C layout often brings a great deal of inconvenience and loss, and even leads to mobilization failure. Under the restriction of mobilization time, the paper establishes the layout model that one or more mobilization logistics centers provide the material to the mobilization demanding place. The mobilization goods or service can reach the demanding place to carry into mobilization execution within the given time, and the number of the built NEM LC s is the least.
\end{abstract}

Index Terms-Center Layout; set covering problem; national economy mobilization; Layout Model; Layout Network diagram

\section{INTRODUCTION}

It is a very important task to use scientific and reasonable method to determine the national economy mobilization logistics center (hereinafter referred to mobilization logistics center). Reasonable layout is to transport the mobilization materials from the NEMLCs to the demanding place by using the least transport capacity, the shortest distance, minimal cost, the least link and the fastest speed. When distributing the NEMLCs, the material supplying to the mobilization demanding point in time was the most important problem which the mobilization host considers. NEMLCs are able to provide the right guarantee from the right place at the right time. This also puts forward the stringent mobilization time to the mobilization logistics system. If the mobilization time is too long, it may lose the significance of mobilization implementation when the mobilization materials shipped to the demand place. Therefore, the study on the layout of NEMLCs has a major economic, social and military

Supported by "the Fundamental Research Funds for the Central Universities"

Sponsored by National Natural Science Foundation of China (NSFC) 71071054 significance. In practice, due to the urgency of mobilization demand, it sometimes may not meet the requirements of mobilization time when building only one mobilization logistics center. Also, setting up only one mobilization logistics center in the large area range is neither consistent with economic principle, nor is the principle of time limit. Therefore, several mobilization logistics center should be built.

During the distribution of mobilization logistics centers, each NEMLC can be viewed as a saving point. In response to war or crisis event, one or more NEMLCs of the region fulfill the functions of mobilization implementation. In general, the more the NEMLCs involved in the implementation, the shorter the mobilization time, and the faster the speed. However, during the process of mobilization implementation, each NEMLC is a mobilization of insecure factor. Especially during the war process, the NEMLC may be enemy targets as the main suppliers of mobilization material. The less saving point may result in the long mobilization time and insufficient material supply. Saving can not have blind spots, so in the time constraints, for any emergency place, there should have at least one to reach within the time limit. On the other hand, when too many NEMLCs are build in the region, some NEMLCs may have not mobilization task. So, these will inevitably result in not only the waste of pertinent resources which mainly include transport, storage, and information, but also huge waste of construction costs and management costs of the NEMLC. Therefore, when distributing the NEMLCs, mobilization host should consider all circumstances synthetically, so that results of the NEMLCs layout can satisfy the needs of mobilization material, but also the economic of mobilization cost.

\section{LAYOUT MODEL}

The maximum characteristic of mobilization demand is the time urgency, when mobilization demand happens, mobilization goods or service are always expected to be conveyed from NEMLC to the mobilization demand site as soon as possible. So time becomes the main optimization goal of the NEMLC layout. During the layout of the NEMLC, the mobilization demand site can 
be treated as the vertex of network graph, the connection line(arc) between vertex and vertex as the distance between two points, but also can be seen as the time required when the speed is constant. Vertex weights, $h_{i}$, stands for the frequency of mobilization requirements. So, the layout problem of the entire NEMLC can be abstracted as an undirected weighted graph.

Given an undirected continuous network graph $G=(V$, $E$ ), which $V$ is non-empty vertex set of graph $G$, and $E$ is the arc set that connecting the vertex.

$$
\begin{aligned}
& V=\left\{v_{1}, v_{2}, L, v_{n}\right\} \\
& E=\left\{e_{1}, e_{2}, L, e_{n}\right\}
\end{aligned}
$$

$h_{i}$ is the weight of vertex $v_{i}, b\left(e_{i}\right)$ is the length of arc $e_{i}$. If the arc $e_{i}$ connects the vertex $v_{p}$ and $v_{q}$, then the arc $e_{i}$ can be expressed as $b\left(e_{i}\right)=b\left(v_{p}, v_{q}\right)$. The shortest path between any two points $x, y$ of $G$ can also be expressed as $d(x, y)$.

Reference [1] illustrates the general one center location problem and reference [2] illustrates the optimal locations for a class of nonlinear single-facility location problems on a network. According to the two reference, when building a NEMLC in the network diagram $G$, the shortest distance between point $\mathrm{X}$ which lies in the $\operatorname{arc} \mathrm{e}_{i}=$ $b\left(v_{p}, v_{q}\right)$ and vertex $v_{i}$ is defined as the distance from point to vertex which can be expressed as $d\left(v_{i}, x\right)$. During the NEMLC layout, the maximum value between point $X$ and each vertex is expected to be the least. Thus, the optimal model can be achieved and be expressed as in

$$
\begin{aligned}
& \min \max \underset{1 \leq i \leq n}{d\left(v_{i}, x\right)} \\
& \text { s.t. } \quad x \in G
\end{aligned}
$$

one of the mathematical expression of network diagram $G$ is distance matrix (time matrix). The distance matrix includes all of the network structure and numerical information which can be used as the starting point to solve the network diagram calculation. The minimum distance matrix is the optimal result of the distance matrix. The advantage of using minimum distance matrix to analyses network problems is that $\mathrm{P}$-center problem and set covering problems can be unified in a platform for discussion. For a given network diagram which has $n$ vertex, the distance matrix $\mathrm{R}$ can be obtained as follows:

$R=\begin{array}{cccccc}0 & r\left(v_{1}, v_{2}\right) & L & r\left(v_{1}, v_{j}\right) & L & r\left(v_{1}, v_{n}\right) \\ r\left(v_{2}, v_{1}\right) & 0 & L & r\left(v_{2}, v_{j}\right) & L & r\left(v_{2}, v_{n}\right) \\ M & M & 0 & L & L & L \\ r\left(v_{i}, v_{1}\right) & r\left(v_{i}, v_{2}\right) & M & r\left(v_{i}, v_{j}\right) & L & r\left(v_{i}, v_{n}\right) \\ M & M & M & M & 0 & L \\ r\left(v_{n}, v_{1}\right) & r\left(v_{n}, v_{2}\right) & M & r\left(v_{n}, v_{j}\right) & M & 0\end{array}$

The element $r\left(v_{i}, v_{j}\right)$ means the distance between the two vertexes $V_{i}$ and $V_{j}$ when there is direct connection, while $r\left(v_{i}, v_{j}\right)=\infty$ when there is no direct connection between the two points. Matrix $R$ is a symmetric matrix.
By the distance matrix $R$, the minimum distance matrix $S$ and the shortest path matrix $P$ can be obtained using Floyd algorithm:

$$
S=\left[\begin{array}{cccccc}
0 & d\left(v_{1}, v_{2}\right) & L & d\left(v_{1}, v_{j}\right) & L & d\left(v_{1}, v_{n}\right) \\
d\left(v_{2}, v_{1}\right) & 0 & L & d\left(v_{2}, v_{j}\right) & L & d\left(v_{2}, v_{n}\right) \\
M & M & 0 & L & L & L \\
d\left(v_{i}, v_{1}\right) & d\left(v_{i}, v_{2}\right) & M & d\left(v_{i}, v_{j}\right) & L & d\left(v_{i}, v_{n}\right) \\
M & M & M & M & 0 & L \\
d\left(v_{n}, v_{1}\right) & d\left(v_{n}, v_{2}\right) & M & d\left(v_{n}, v_{j}\right) & M & 0
\end{array}\right]
$$

The element $d\left(v_{i}, v_{j}\right)$ means the shortest distance between the two vertexes $v_{i}$ and $v_{j}$. The minimum distance matrix is also a symmetric matrix.

$$
P=\left[\begin{array}{cccccc}
0 & p\left(v_{1}, v_{2}\right) & L & p\left(v_{1}, v_{j}\right) & L & p\left(v_{1}, v_{n}\right) \\
p\left(v_{2}, v_{1}\right) & 0 & L & p\left(v_{2}, v_{j}\right) & L & p\left(v_{2}, v_{n}\right) \\
M & M & 0 & L & L & L \\
p\left(v_{i}, v_{1}\right) & p\left(v_{i}, v_{2}\right) & M & p\left(v_{i}, v_{j}\right) & L & p\left(v_{i}, v_{n}\right) \\
M & M & M & M & 0 & L \\
p\left(v_{n}, v_{1}\right) & p\left(v_{n}, v_{2}\right) & M & p\left(v_{n}, v_{j}\right) & M & 0
\end{array}\right]
$$

The element $p\left(v_{i}, v_{j}\right)$ means the shortest path between the two vertexes $V_{i}$ and $V_{j}$. Sometimes the shortest path is not exclusive.

During the planning of NEMLC, due to the characteristic of NEMLC, some enterprises in the region can be acted as candidate mobilization logistics center so as to fulfill the function of transferring civil use to military use. One or more mobilization logistics centers can be selected from the known place. First the model focuses only one place that can be selected.

\section{A. One Mobilization Logistics Center Layout}

Because the NEMLC is selected from the known place, one idea is the candidate mobilization logistics center can cover more mobilization demand places within the given time, and another idea is that the candidate mobilization logistics center can reach the mobilization demand places in the shortest time.

Taking the first condition into consideration, the NEMLC is built when the candidate mobilization logistics center can cover more mobilization demand places within the given time.

Define a matrix $A=\left(a_{i j}\right)_{m \times n}$, and suppose the mobilization time as $T$. Then

$$
a_{i j}= \begin{cases}1 & t_{i j} \leq T \\ 0 & t_{i j}>T\end{cases}
$$

So the shortest time matrix $S$ can be transformed into the coefficient matrix $A$. 
$\mathrm{a}_{\mathrm{ij}}=1$ means that the candidate mobilization logistics center $V_{i}$ can reach mobilization demand place $V_{j}$ and provide the mobilization materials to $\mathrm{V}_{\mathrm{j}}$. That is to say, vertex $V_{i}$ can cover $V_{j}$ within the given time, or $V_{i}$ is a covering of $V_{j}$. While $a_{i j}=0$ means $V_{i}$ can not provide the mobilization materials to $V_{j}$ within the given time.

Take the network structure Fig. 1 for instance, and suppose the nine vertexes are the candidate mobilization logistics center, and mobilization demand places at the same time. Now choose a vertex as the mobilization logistics center. First set the constant $h_{i}$ as 1 , and given the mobilization time $\mathrm{T}=10.5$.

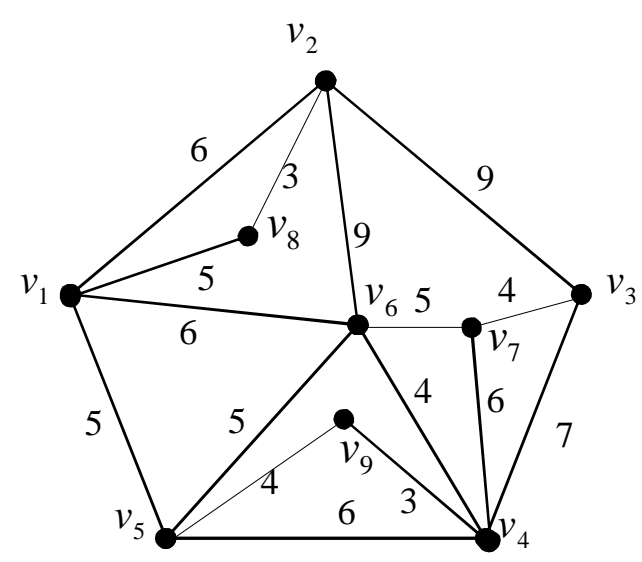

Figure 1. network layout figure of NEMLC

The minimum distance matrix $\mathrm{S}$ can be obtained using Floyd algorithm. Then, transform the shortest time matrix $S$ into the coefficient matrix $A$.

$$
S=\left[\begin{array}{ccccccccc}
0 & 6 & 15 & 10 & 5 & 6 & 11 & 5 & 9 \\
6 & 0 & 9 & 13 & 11 & 9 & 13 & 3 & 15 \\
15 & 9 & 0 & 7 & 13 & 9 & 4 & 12 & 10 \\
10 & 13 & 7 & 0 & 6 & 4 & 6 & 15 & 3 \\
5 & 11 & 13 & 6 & 0 & 5 & 10 & 10 & 4 \\
6 & 9 & 9 & 4 & 5 & 0 & 5 & 11 & 7 \\
11 & 13 & 4 & 6 & 10 & 5 & 0 & 16 & 9 \\
5 & 3 & 12 & 15 & 10 & 11 & 16 & 0 & 14 \\
9 & 15 & 10 & 3 & 4 & 7 & 9 & 14 & 0
\end{array}\right]
$$

$$
A=\left[\begin{array}{lllllllll}
1 & 1 & 0 & 1 & 1 & 1 & 0 & 1 & 1 \\
1 & 1 & 1 & 0 & 0 & 1 & 0 & 1 & 0 \\
0 & 1 & 1 & 1 & 0 & 1 & 1 & 0 & 1 \\
1 & 0 & 1 & 1 & 1 & 1 & 1 & 0 & 1 \\
1 & 0 & 0 & 1 & 1 & 1 & 1 & 1 & 1 \\
1 & 1 & 1 & 1 & 1 & 1 & 1 & 0 & 1 \\
0 & 0 & 1 & 1 & 1 & 1 & 1 & 0 & 1 \\
1 & 1 & 0 & 0 & 1 & 0 & 0 & 1 & 0 \\
1 & 0 & 1 & 1 & 1 & 1 & 1 & 0 & 1
\end{array}\right]
$$

From the above analysis, we can see that within the given time, the more the vertex covers other vertexes, the better establishes the mobilization logistics center at that vertex. Suppose the covering of the vertex $V_{i}$ to other vertexes is represented by $N_{i}$. According to the coefficient matrix:

$$
\begin{aligned}
& N_{1}=\{1,2,4,5,6,8,9\}, \\
& N_{2}=\{1,2,3,6,8\}, \\
& N_{3}=\{2,3,4,6,7,9\}, \\
& N_{4}=\{1,3,4,5,6,7,9\}, \\
& N_{5}=\{1,4,5,6,7,8,9\}, \\
& N_{6}=\{1,2,3,4,5,6,8,9\}, \\
& N_{7}=\{3,4,5,6,7,9\}, \\
& N_{8}=\{1,2,5,8\}, \\
& N_{9}=\{1,3,4,5,6,7,9\} .
\end{aligned}
$$

Obviously, the maximum value is obtained in the vertex $V_{6}$, where $\left|N_{6}\right|=8$. So, NEMLC is built in the vertex $\mathrm{V}_{6}$, as the star shown in Fig. 2.

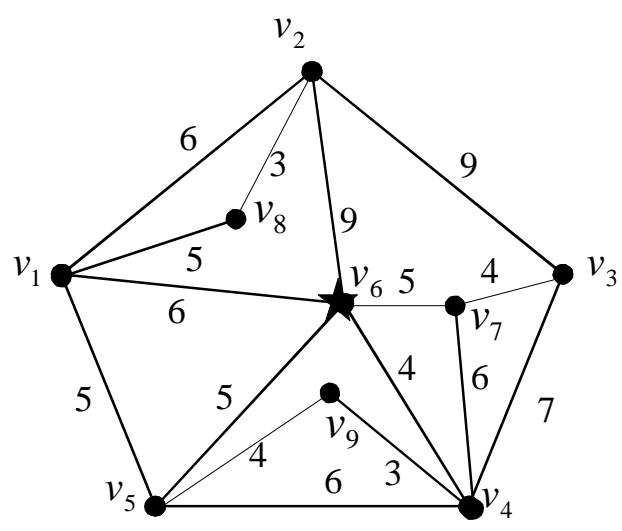

Figure 2. NEMLC layout when covering more demand site

As a matter of fact, the frequency of each mobilization demand site is usually not entirely the same. For example, the probability of emergency happening at the remote mountain area or rural area is relatively low, while quite a lot higher in modern prosperous city.

If frequency coefficient $h_{i}$ is not 1 , the model (1) can transfer to model (3). 


$$
\begin{aligned}
& \min \sum_{i=1}^{n} h_{i} d\left(v_{i}, x\right) \\
& \text { s.t. } \max _{1 \leq i \leq n} d\left(v_{i}, x\right) \leq T \\
& x \in G
\end{aligned}
$$

Solving the problem, in fact, is to search the absolute median point of the network $G$. That is, the point that make the $\sum_{i=1}^{n} h_{i} d\left(v_{i}, x\right)$ least, and the vertex $v_{5}$ is exactly that point by calculation, as the star shown in Fig. 3.

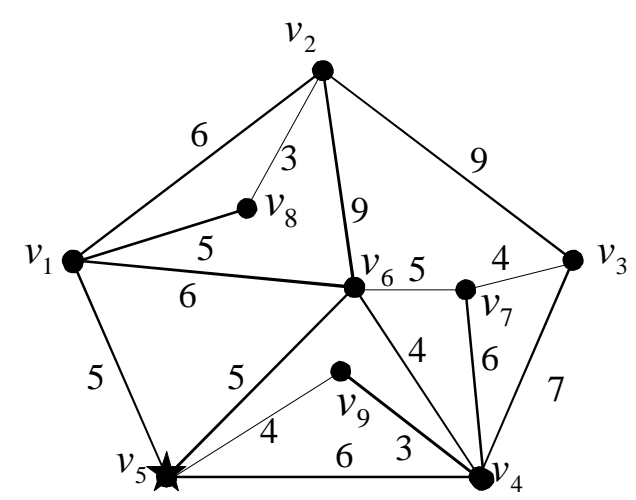

Figure 3. NEMLC layout model

But when the mobilization time $T=10.5$, and $h_{i}=$ $(4,2,2,3,5,3,3,6,5)$, the covering when building the NEMLC at the vertex $v_{5}$ is $\left|N_{5}\right|=7$, while the covering when building the NEMLC at the vertex $\mathrm{V}_{6}\left|\mathrm{~N}_{6}\right|=8$. So, the decision maker should decide to build the NEMLC at the vertex $v_{5}$ or $v_{6}$ under the consideration of all the circumstances. Of course, the ideal situation is that within a given time, all the vertexes can be covered. So more mobilization logistics centers should be built, which will be considered next.

Taking the second condition into consideration, that is, the candidate mobilization logistics center can reach the mobilization demand places in the shortest time.

Still take the network structure figure 1 for instance, and suppose the nine vertexes are the candidate mobilization logistics center, and mobilization demand places at the same time. Now choose a vertex as the mobilization logistics center.

Because NEMLC is distributed at the vertex of the network diagram which is also a known place, the solution of the problem, in fact, is the absolute median point of the network diagram $G$. when the frequency coefficient $h_{i}$ is the constant 1 , the solution location is in the vertex $v_{6}, \sum_{i=1}^{n} h_{i} d\left(v_{i}, v_{6}\right)=56$, the absolute radius $r\left(x_{m}\right)=11$. That is, if the mobilization time $T$ is less than 11 , build a mobilization logistics center in the vertex $\mathrm{V}_{6}$ can meet the needs of mobilization demand places. The solution diagram is the same as the Fig. 2.
If frequency coefficient $h_{i}$ is not 1 , still set the value $h_{i}=(4,2,2,3,5,3,3,6,5)$, the optimal solution is in the vertex $V_{5}$ where the absolute median radius $r\left(X_{m}\right)=13$, That is, if the mobilization time $T$ is less than 13 , build a mobilization logistics center in he vertex $\mathrm{V}_{6}$ can meet the needs of mobilization demand places.

\section{B. Several Mobilization Logistics C enter Layout}

In the practice of mobilization implementation, the condition often encountered is that a number of NEMLCs provide mobilization material when mobilization time is given. The following discussion is focused on such layout model.

This is a typical set covering problem. The basic wayout is: firstly find the point set that can satisfy the regional coverage restriction, and then find the optimal point that can meet the demand of the condition in the set point. Set covering problem has been widely applied to aviation personnel scheduling, circuit design, vehicle routing, and so on. The problem has been proved to be an NP-complete problem. Reference [3] illustrates set covering by single-branch enumeration with linearprogramming subproblems.

Set Covering Problem is one of the classical combinatorial optimal ones the same as a NP difficult problem which is mainly solved by the heuristic algorithm. Reference [4] dual-based heuristics for a hierarchical covering location problem, reference [5] introduces a heuristic algorithm of solving the problem of set covering problem, reference [6] puts forward the heuristic function algorithm of set covering problem, while reference [7] presents a maximum expected covering location problem: Formulation, properties, and heuristic solution.

Heuristic method is a method that approximates to the optimal solution step by step. Exploited at the end of 1950s, heuristic function algorithm of set covering problem can be described as follows:

(1) $\mathrm{R}=\mathrm{M}, \mathrm{S}=\phi, \mathrm{t}=1$;

(2) if $\mathrm{R}=\phi$, then turn ( 4$)$, else, set $K_{j}=\left|M_{j} I R\right|(j \in N)$, select $j(t)$ to the effect that $f\left(C_{j(t)}, K_{j}\right)=\min _{K_{j}>0}\left(C_{j}, K_{j}\right) \quad, \quad R=R-M_{j(t)}$, $S=S \cup\{j(t)\}$, in which $M_{j}=\left\{i \in M \mid a_{i j}=1\right\}$;

(3) $\mathrm{t}=\mathrm{t}+1$, turn (2) ;

(4) Rank the elements of the $S$ in descending order, consider $i \in S$ successively, if $S-\{i\}$ is feasible solution, then set $S-\{i\}$. When all the elements of $S$ are considered, then $\mathrm{S}$ is the optimal solution of the problem.

Common forms of the heuristic function of $f\left(c_{j(t)}, K_{j}\right)$ in Step (2) are listed as follows:
(1) $c_{j}$;
(2) $\mathrm{C}_{\mathrm{j}} / \mathrm{K}_{j}$; 

(3) $c_{j} /\left(\log _{2}^{k}\right)$;
(4) $c_{j} /\left(K_{j} \log _{2}^{k}\right)$;
(5) $c_{j} /\left(K_{j} \ln K_{j}\right)$;
(6) $c_{j} / K^{2}$;
(7) $\left(C_{j}\right)^{1 / 2} / K_{j}^{2}$;

Suppose $F=\left\{F_{1}, F_{2}, L, F_{m}\right\}$ is the demand site set for the mobilization, $S=\left\{S_{1}, S_{2}, L, S_{n}\right\}$ is the NEMLCs site set to be selected, $F_{i}(i=1,2, L, m)$ is the mobilization demand site, $S_{j}(j=1,2, L, n)$ is the NEMLC site to be selected, $t_{i j}$ is the minimum time between the NEMLC $S_{j}$ to be selected and the mobilization demand place $F_{i}$.

How to select and build NEMLC from the NEMLCs site set to be selected $S=\left\{S_{1}, S_{2}, L, S_{n}\right\}$, ensure that when any mobilization demand site $\mathrm{F}=\left\{\mathrm{F}_{1}, \mathrm{~F}_{2}, \mathrm{~L}, \mathrm{~F}_{\mathrm{m}}\right\}$ appears emergency, at least one or more NEMLC can reach the mobilization demand site and meet the needs of the site, besides, the number of the NEMLCs is the least.

For the problem above, define a matrix $A=\left(a_{i j}\right)_{m \times n}$, and suppose the mobilization time as $\mathrm{S}$. Then

$$
a_{i j}= \begin{cases}1 & t_{i j} \leq s \\ 0 & t_{i j}>s\end{cases}
$$

$a_{i j}=1$ stands for that the NEMLCs site to be selected $S_{j}$ can reach the mobilization demand site $F_{i}$ within the given time, while stands for that $S_{j}$ can not reach $F_{i}$ within the given time.

Definition 1: For any $\mathrm{S}_{j} \in \mathrm{S}$, $N\left(S_{j}\right)=\left\{F_{i} \mid a_{i j}=1, i=1,2, L, m\right\}$ is called the mobilization object set of NEMLC $S_{j}$.

Definition 2: For any $F_{i} \in F$, $N_{i}=\left\{j \mid t_{i j} \leq s, j=1,2, L, n\right\}$ is called the NEMLC set to the mobilization demand site $F_{i}$.

Definition 3: Suppose $\mathrm{J}=\{1,2, \mathrm{~L}, \mathrm{n}\}$, if $\mathrm{J}^{*} \subseteq \mathrm{J}$, and $\bigcup_{j \in J^{*}} N\left(S_{j}\right)=F$, then $J^{*} \operatorname{covers} F$, or $J^{*}$ as a covering of $F$.

Given $\mathrm{J}^{*} \subseteq \mathrm{J}$, define $X=\left\{X_{1}, X_{2}, L, X_{n}\right\}^{T}$, which

$$
X_{j}= \begin{cases}1 & j \in J^{*} \\ 0 & j \notin J^{*}\end{cases}
$$

Conversely, if given $X=\left\{X_{1}, X_{2}, L, X_{n}\right\}^{T}$, then the set $\mathrm{J}^{*}=\left\{\mathrm{j} \mid \mathrm{X}_{\mathrm{j}}=1,1 \leq \mathrm{j} \leq \mathrm{n}\right\} \subseteq \mathrm{J}$ can be defined.
Suppose at least $b_{i}$ NEMLCs can reach the mobilization demand place $F_{i}$ and carry on the mobilization implementation within the given time. Because of the actual situation difference of each mobilization demand place, the problem model can be expressed as the following:

$$
\begin{array}{ll}
\min z=\sum_{j=1}^{n} c_{j} X_{j} & \\
\text { s.t. } \sum_{j=1}^{n} a_{i j} X_{j} \geq b_{i} & i \in M=\{1,2, L, m\} \\
X_{j} \in\{0,1\} & j \in N=\{1,2, L, n\}
\end{array}
$$

among which $C_{j}$ stands for the cost of building NEMLC in $S_{j}$.

$$
X_{j}=\left\{\begin{array}{l}
1 \\
\text { if bulid NEMLC in } S_{j} \\
0 \text { if not bulid NEMLC in } S_{j}
\end{array}\right.
$$

Solving the model is to find a NEMLC set which can cover all mobilization demand place. Set Covering Problem is a NP difficult problem which is mainly solved by the heuristic algorithm[3-7]. The main algorithm for solving this model is described as following:

(1) $R=M, S=\phi, t=1, N_{i}=\left\{j \mid j \in N, a_{i j}=1\right\},(i \in M)$;

(2) $\exists i \in M,\left|N_{i}\right|<b_{i}$, no feasible solution, end, otherwise turn (3) ;

(3) For $i \in M$, if $\left|N_{i}\right|=b_{i}$, then $\forall j \in N_{i}, X_{j}=1$, $\mathrm{S}=\mathrm{S} \cup\{j\}$

(4) $b_{i}=b_{i}-a_{i j}$, if $b_{i} \leq 0$, then $R=R-\{i\}$, for all $i \in R$, calculate $\left|N_{i}\right|=\left|N_{i}\right|-a_{i j}$;

(5) if $R=\phi$, turn (7), otherwise turn (6) ;

(6) Select $j(t), f(j(t))=\max _{j \in N-S}\left\{\frac{1}{c_{j}} \sum_{i \in R} b_{i} a_{i j} /\left|N_{i}\right|\right\}$, then $\mathrm{S}=\mathrm{S} \cup\{\mathrm{j}(\mathrm{t})\}, \mathrm{t}=\mathrm{t}+1$, turn (4) ;

(7) Rank the element $C_{j}$ of the $S$ sort descending, take out $j \in S$ in turn, if for all $i \in M, b_{i}+a_{i j}<1$, then $b_{i}=b_{i}+a_{i j}, S=S-\{j\}$, so $S$ is the best solution of the problem.

Also, take the network structure Fig. 1 for instance.

Suppose all mobilization time limit $\mathrm{s}$ are 9 , and the number of units of the edge means transport time. When a vertex emergency appears, the number of NEMLC carrying on the mobilization implementation within the given time is $b=\{3,3,3,2,3,4,2,3,3\}$. Respectively, the construction cost of each vertex is $C=\{12,15,17,11,14,18,23,13,28\}$, because of the different location. Thus, the shortest time matrix $S$ can be gotten by Floyd algorithm. 


$$
\mathrm{S}=\left[\begin{array}{ccccccccc}
0 & 6 & 15 & 10 & 5 & 6 & 11 & 5 & 9 \\
6 & 0 & 9 & 13 & 11 & 9 & 13 & 3 & 15 \\
15 & 9 & 0 & 7 & 13 & 9 & 4 & 12 & 10 \\
10 & 13 & 7 & 0 & 6 & 4 & 6 & 15 & 3 \\
5 & 11 & 13 & 6 & 0 & 5 & 10 & 10 & 4 \\
6 & 9 & 9 & 4 & 5 & 0 & 5 & 11 & 7 \\
11 & 13 & 4 & 6 & 10 & 5 & 0 & 16 & 9 \\
5 & 3 & 12 & 15 & 10 & 11 & 16 & 0 & 14 \\
9 & 15 & 10 & 3 & 4 & 7 & 9 & 14 & 0
\end{array}\right]
$$

According to the mobilization time limit, the shortest time matrix $S$ can be transformed into the coefficient matrix $A$ :

$$
A=\left[\begin{array}{lllllllll}
1 & 1 & 0 & 0 & 1 & 1 & 0 & 1 & 1 \\
1 & 1 & 1 & 0 & 0 & 1 & 0 & 1 & 0 \\
0 & 1 & 1 & 1 & 0 & 1 & 1 & 0 & 0 \\
0 & 0 & 1 & 1 & 1 & 1 & 1 & 0 & 1 \\
1 & 0 & 0 & 1 & 1 & 1 & 0 & 0 & 1 \\
1 & 1 & 1 & 1 & 1 & 1 & 1 & 0 & 1 \\
0 & 0 & 1 & 1 & 0 & 1 & 1 & 0 & 1 \\
1 & 1 & 0 & 0 & 0 & 0 & 0 & 1 & 0 \\
1 & 0 & 0 & 1 & 1 & 1 & 1 & 0 & 1
\end{array}\right]
$$

Because the mobilization demand place is also the candidate location of NEMLC, for the sake of distinction, $F$ stands for the vertex when it acts as a mobilization demand place, while $S$ stands for the vertex when it acts as a NEMLC. Detailed calculation steps are as follows:

(1) $\mathrm{R}=\mathrm{M}=\{1,2,3,4,5,6,7,8,9\} \quad, \quad \mathrm{S}=\phi$

$\mathrm{t}=1 ; \mathrm{N}_{1}=\{1,2,5,6,8,9\}, \mathrm{N}_{2}=\{1,2,3,6,8\}, \mathrm{N}_{3}=\{2,3,4,6,7\}$, $\mathrm{N}_{4}=\{3,4,5,6,7,9\} \quad, \quad \mathrm{N}_{5}=\{1,4,5,6,9\}$

$\mathrm{N}_{6}=\{1,2,3,4,5,6,8,9\} \quad, \quad \mathrm{N}_{7}=\{3,4,6,7,9\}, \quad \mathrm{N}_{8}=\{1,2,8\}$, $\mathrm{N}_{9}=\{1,4,5,6,7,9\}$;

(2) $\exists i \in M,\left|N_{i}\right| \geq b_{i}$, turn (3) ;

(3) $\left|N_{8}\right|=b_{8}$, then $X_{1}=X_{2}=X_{8}=1, S=\{1,2,8\}$;

(4) $b_{1}=0, b_{2}=0, b_{3}=1, b_{4}=2, b_{5}=2, b_{6}=1$, $b_{7}=2, \quad b_{8}=0, b_{9}=2 . R=\{3,4,5,6,7,9\},\left|N_{3}\right|=4$, $\left|N_{4}\right|=6,\left|N_{5}\right|=4,\left|N_{6}\right|=5,\left|N_{7}\right|=5,\left|N_{9}\right|=5$;

(5) $\mathrm{R} \neq \phi$, turn (6) ;

(6) Calculate $f(j(1))=\max _{j \in\{3,4,5,6,7,9\}}\left\{\frac{1}{c_{j}} \sum_{i \in R} b_{i} a_{i j} /\left|N_{i}\right|\right\}$, by calculating, when $\mathrm{j}(1)=4, \mathrm{f}(\mathrm{j}(1))=0.35$, obtain maximum value, so select $\mathrm{j}(1)=4$, then $S=\{1,2,8,4\}$, $\mathrm{t}=2$;

(7) Calculate and obtain $b_{1}=0, b_{2}=0, b_{3}=0$, $b_{4}=1, b_{5}=1, b_{6}=0, b_{7}=1, b_{8}=0, b_{9}=1, R=\{4,5,7,9\}$, $\left|N_{4}\right|=5,\left|N_{5}\right|=3,\left|N_{7}\right|=4,\left|N_{9}\right|=4$;
(8) $R \neq \phi$, calculate $f(j(2))=\max _{j \in\{3,5,6,7,9\}}\left\{\frac{1}{c_{j}} \sum_{i \in R} b_{i} a_{i j} / N_{i} \mid\right\}$, by calculating, when $\mathrm{j}(2)=6, \mathrm{f}(\mathrm{j}(2))=0.057$, obtain maximum value, so select $j(2)=6$, then $S=\{1,2,8,4,6\}$, $\mathrm{t}=3$;

(9) Calculate and obtain $b_{1}=-1, b_{2}=-1, b_{3}=-1$, $b_{4}=0, b_{5}=0, b_{6}=-1, b_{7}=0, b_{8}=0, b_{9}=0, R \neq \phi$;

(10) Descending the elements of $S$ in order, $S=\{6,2,8,1,4\}$, by inspection, $S=\{6,2,8,1,4\}$, therefore the optimal solution of the model is $X_{1}=X_{2}=X_{4}=X_{6}=X_{8}=1$.

The calculation result is shown in table 1 .

TABLE I. THE CALCULATION RESULT

\begin{tabular}{|c|c|c|c|c|c|c|c|c|c|}
\hline $\begin{array}{c}\text { candidate } \\
\text { location }\end{array}$ & 1 & 2 & 3 & 4 & 5 & 6 & 7 & 8 & 9 \\
\hline cost & 12 & 15 & 17 & 11 & 14 & 18 & 23 & 13 & 28 \\
\hline construction & $\sqrt{ }$ & $\sqrt{ }$ & $\times$ & $\sqrt{ }$ & $\times$ & $\sqrt{ }$ & $\times$ & $\sqrt{ }$ & $\times$ \\
\hline
\end{tabular}

Therefore, the optimal function value of the model is $z^{*}=69$, which NEMLCs are built in the location $1,2,4,6$, 8, as the star shown in Fig. 4.

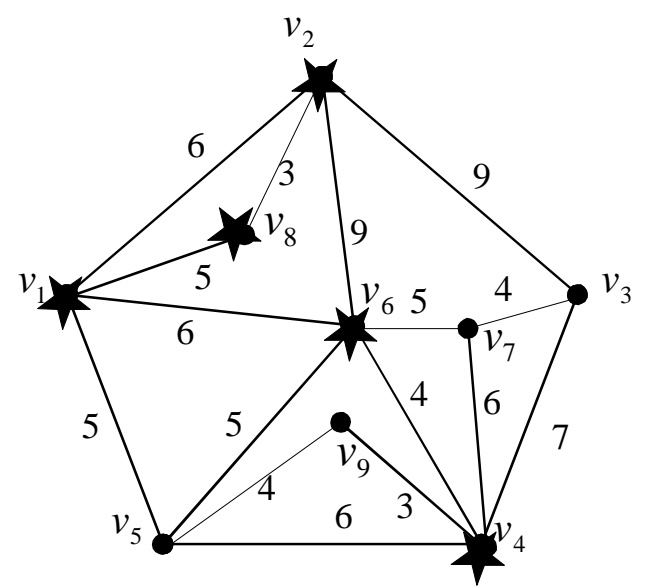

Figure 4. network layout result figure of NEMLCs

In this model only the minimum number of NEMLCs is considered, but the difference of actual construction costs fails to be taken into account. The model and the algorithm have some practical significance in the city region position when the construction cost of the NEMLCs is nearly the same.

Layout problem of NEMLCs is one of the most important long-term decision-making. The layout result will have a direct impact on the saving time, reliability, mobilization quality, mobilization efficiency, mobilization costs, etc, thus affecting the mobilization effect. Reasonable NEMLCs layout can bring people convenience in the daily life, reduce costs, and improve service efficiency and competitiveness. Thus, NEMLCs can gather all kinds of mobilization materials and objects quickly to meet the mobilization needs in the emergency or wartime, and recover its original functions rapidly after 
the termination of the mobilization task. Poor NEMLCs layout often brings a great deal of inconvenience and loss, and even leads to mobilization failure.

\section{ACKNOWLEDGMENT}

My sincere gratitude goes primarily to Professor Kong Zhaojun, my supervisor in school of management and economics, Beijing Institute of Technology, for his constant encouragement and guidance. He has walked me through all the stages of the writing of this paper. Without his consistent and illuminating instruction, this paper could not have reached its present form.

Second, I would like to express my heartfelt appreciation to the professors and teachers at School of Economics and Management, North China Electric Power University. They have instructed and helped me a lot in writing this paper. I also owe my thankfulness to my friends and my fellow classmates who helped me work out my problems during the difficult course of this paper.

\section{REFERENCES}

[1] Roland Durier. The general one center location problem. Mathematics of Operations Research, 1995, 20(2): 400-418.

[2] Shier D.R, Dearing P.M. Optimal locations for a class of nonlinear single-facility location problems on a network [J] Operations Research. 1983, 31: 292-303.

[3] Lemke C E. Set Covering by Single-Branch Enumeration with Linear-Programming Subproblems [J]. Operations Research, 1971, 19(4): 998-1022.

[4] Luis Gonzalo Acosta Espejo, Roberto D.Galvao. Dualbased heuristics for a hierarchical covering location problem[J]. Computers \& Operations Research. 2003, 30: 65-180.

[5] Chen Duanbing, Huang Wenqi. A heuristic algorithm of Solving the problem of set covering problem[J], Computer Science, 2007, 34 (4): 133-136.

[6] Quan Guangri, Hong Bingrong, Ye Feng, et al. The heuristic function algorithm of set covering problem[J], Journal of Software, 1998 (2): 156-160.

[7] Daskin M S. A maximum expected covering location problem: Formulation, properties, and heuristic solution[J]. Transportation Service.1983, 17: 48-70.

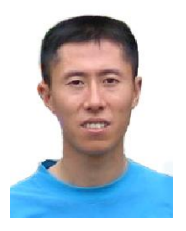

Tang. Pingzhou, was borned on may 26, 1973, in TangShan city, HeBei province.

Got the Bachelor's Degree in Mechanics field on july, 1996, at North China Electric Power University, Baoding, Hebei province. Got the Master's Degree in Mechanics and Electronic field, at North China Electric Power University, Beijing, on April, 2000. Got the Doctor's Degree in management field, at Beijing Institute of Technology, Beijing, on June, 2010. The major field of study includes national economy mobilization, information management and decision support, mobilization logistics, and so on.

He worked at Sino-Italy Joint Venture TaiMa Sanitary Company, TangShan city, Hebei provice. Now he worked in NORTH CHINA ELECTRIC POWER UNIVERSITY, Beijing. His publications listed as follows: [1] Layout of National Economic Mobilization Logistics Centers, Beijing, 2010. [2] The research on BP neural network model based on guaranteed convergence particle swarm optimization, IITA2008 11. [3] The Evaluation Study on the Ability of National Economy Mobilization Logistics Center based on Fuzzy-Grey Comprehensive Evaluation Method, WICOM, 2008.10. His research interests refer to logistics layout model and algorithm, theory and method of information management and decision support, and so on.

Tian. Huiying, was borned on may, 1967, in Baoding city, HeBei province.

Got the Bachelor's Degree in dynamic engineering field on july, 1989, at North China University of Water Resources and Electric Power, Handang, Hebei province. The major field of study includes information management and decision support, electronic commerce, and so on.

She worked in NORTH CHINA ELECTRIC POWER UNIVERSITY, Beijing. Her publications listed as follows: [1] Study on location selection of national economy mobilization logistics center based on fuzzy-grey comprehensive evaluation method, WICOM, 2008.10. [2] On optimum reliability management for generation systems, POWERCON 1998. [3] Construction and Methodology of Comprehensive Evaluation System for Credit of Industrial Electricity Customers, Power System Technology, 2007.1. Her research interests refer to theory and method of information management and decision support, and so on. 